Research Paper

\title{
High viral loads of circulating Epstein-Barr virus DNA copy number in peripheral blood is associated with inferior prognosis in patients with mantle cell lymphoma
}

\author{
Xiao-Hui Zhou ${ }^{1,2,3 *}$, Jin-Hua Liang1,2,3*, Li Wang1,2,3, Hua-Yuan Zhu'1,2,3, Jia-Zhu Wu Wu $^{1,2,3}$, Yi Xia ${ }^{1,2,3}$, Yue \\ $\operatorname{Li}^{1,2,3}$, Shu-Chao Qin 1,2,3, Lei Fan ${ }^{1,2,3}$, Jian-Yong Li1,2,3, Wei Xu1,2,3凶 \\ 1. Department of Hematology, the First Affiliated Hospital of Nanjing Medical University, Jiangsu Province Hospital, Nanjing 210029, China \\ 2. Key Laboratory of Hematology of Nanjing Medical University, Nanjing 210029, China. \\ 3. Collaborative Innovation Center for Cancer Personalized Medicine, Nanjing 210029, China. \\ *These authors contributed equally to this work.
}

$\triangle$ Corresponding author: Dr Wei Xu, Department of Hematology, the First Affiliated Hospital of Nanjing Medical University, Jiangsu Province Hospital, Nanjing 210029, China; Key Laboratory of Hematology of Nanjing Medical University, Nanjing 210029, China; Collaborative Innovation Center for Cancer Personalized Medicine, Nanjing 210029, China. Telephone: +86-25-83781120; Fax: +86-25-83781120; E-mail: xuwei10000@hotmail.com.

(C) The author(s). This is an open access article distributed under the terms of the Creative Commons Attribution License (https://creativecommons.org/licenses/by/4.0/). See http://ivyspring.com/terms for full terms and conditions.

Received: 2019.06.10; Accepted: 2019.12.09; Published: 2020.06.21

\begin{abstract}
Mantle cell lymphoma $(\mathrm{MCL})$ is a distinct subtype of $B$ cell non-Hodgkin lymphoma. No research has yet documented to investigate the prognostic implications of Epstein-Barr virus (EBV) infection in MCL. The objective of this study was to determine whether EBV DNA load may influence the heterogeneity in the course of the disease in MCL patients. Eighty-eight MCL patients were retrospectively enrolled in the study. EBV DNA load was detected by real-time quantitative PCR for quantification. The univariate and multivariate Cox proportional hazards models were established for the estimation of prognostic factors. Twenty-seven patients were detected positive for EBV DNA and the median virus titer was $1.72 \times 10^{4}$ copies $/ \mathrm{mL}$ (range, $8.20 \times 10^{2}$ to $4.14 \times 10^{5}$ copies $/ \mathrm{mL}$ ). With a median follow-up of 39 months (range, 9 to 120 months), patients in EBV DNA-positive group displayed unfavorable progression-free survival (PFS) $(P=0.012)$ and overall survival $(O S)(P=0.004)$ than patients in EBV DNA-negative group. Multivariate Cox regression analysis revealed that EBV DNA-positivity was an independent risk factor for both PFS (HR, 2.04; $95 \% \mathrm{Cl}, 1.07$ to $3.92 ; P=0.031$ ) and $\mathrm{OS}(\mathrm{HR}, 2.68 ; 95 \% \mathrm{Cl}, 1.20$ to $6.00 ; P=0.016$ ). Reduction in $\mathrm{EBV}$ copies was significantly associated with therapy-response.

Circulating EBV DNA load in whole blood proved to be a significant predictor of prognosis in patients with MCL, which needs further validation in large-scale clinical studies.
\end{abstract}

Key words: Mantle cell lymphoma; Circulating Epstein-Barr virus DNA load; Overall survival; Progression free survival

\section{Introduction}

Mantle cell lymphoma (MCL) is a distinct subtype of B cell non-Hodgkin lymphoma (NHL) manifested by extensive lymphadenopathy, blood and bone marrow involvement, and splenomegaly with a short remission duration to standard therapies [1]. The past few decades have witnessed great progresses in improving the outcomes of MCL patients owing to the widespread use of rituximab in combination with anthracycline-containing regimens as well as new approaches [2]. However, patients with MCL still presented great heterogeneity in the clinical course with a median overall survival (OS) of 3-5 years [1].

Epstein-Barr virus (EBV), also known as human herpesvirus 4 (HHV-4), is a ubiquitous herpesvirus that infects more than $90 \%$ of all humans, contributing to the development of EBV-associated lymphomas, lymphoproliferative disorders, hemophagocytic 
lymphohistiocytosis and solid tumors [3]. Several researches showed that high level of EBV DNA loads in whole blood or plasma predicted adverse prognosis in EBV-negative lymphomas, such as chronic lymphocytic leukemia (CLL), invariably EBV-positive lymphomas, such as extranodal natural killer/T-cell lymphoma (ENKTL) and angioimmunoblastic T-cell lymphoma (AITL), and partially EBV-positive lymphomas, such as diffuse large B-cell lymphoma (DLBCL) and Hodgkin lymphoma (HL) [4-8]. Though MCL is not currently considered as a subtype of EBV-associated lymphoma, some of the patients are inevitably infected with EBV [9]. Notably, due to the rarity of $\mathrm{MCL}$, the pathogenetic roles and detailed mechanisms of EBV in the development of MCL are dismal. Furthermore, there are no reports on the prevalence and clinical significances of EBV in patients with MCL until now. Hence, we conducted the retrospective analysis to comprehensively explore the potential prognostic impacts of circulating EBV DNA load in 88 MCL patients.

\section{Materials and Methods}

\section{Patients}

A total of 88 consecutive subjects histologically newly diagnosed MCL patients between September 2008 and November 2017 were enrolled in this retrospective study in the First Affiliated Hospital of Nanjing Medical University, Jiangsu Province Hospital (Nanjing, China). Diagnosis of MCL, dependent on a surgical specimen, preferably a lymph node biopsy, was in accordance with criteria of World Health Organization (WHO) classification 2008 [10]. Besides, detection of $t(11 ; 14)(\mathrm{q} 13 ; \mathrm{q} 32)$ and overexpression of cyclin D1 is mandatory for the diagnosis of MCL. Inclusion criteria were as follows: histologically confirmed MCL; at least had one efficacy assessment after receiving first-line chemotherapy of R-hyper-CVAD regimen (rituximab, cyclophosphamide, doxorubicin, vincristine and dexamethasone) alternated with the MA regimen (high-dose methotrexate and cytarabine) or a modified R-hyper-CVAD regimen or R-CHOP-like (rituximab, cyclophosphamide, vincristine, doxorubicin and prednisolone) regimen. Blastoid type of MCL was excluded from this study.

Efficacy assessment was strictly in accordance with International Working Group Criteria and responses were classified as complete response (CR), unconfirmed $\mathrm{CR}(\mathrm{CRu})$, partial response $(\mathrm{PR})$, stable disease (SD), and progressive disease (PD) [11].

\section{Data collection}

Baseline clinical characteristics including gender, age, bone marrow involvement (BMI), Ann Arbor stage, Eastern Cooperative Oncology Group (ECOG) score, B symptoms, white blood cell (WBC) counts, absolute monocyte counts (AMC), serum lactic dehydrogenase (LDH), serum beta- 2 microglobulin level ( $\beta 2-\mathrm{MG})$ and simplified MCL International Prognostic Index (sMIPI) score (covering age, ECOG, LDH and WBC) at admission were entirely available.

EBV DNA load at initial diagnosis, as well as, before each cycle of chemotherapy was detected by real-time quantitative PCR with an ABI PRISM 7500 (Applied Biosystems, Foster City, CA) using DNA extracted with the EBV-PCR Fluorescence Quantitative Diagnostic Kit (Da An Gene Co., Guangzhou, China) from whole blood according to instruction manuals. As for EBV DNA copy number, with the improvement of sensitivity, the boundary of $5 \times 10^{2}$ copies / $\mathrm{mL}$ was established as a cut-off value to distinguish EBV DNA-positive from EBV DNAnegative individuals.

Furthermore, anti-EBV antibody detection, consisting of Epstein-Barr nuclear antigen EBNA-IgG, EBV-VCA-IgG, EBV-VCA-IgA, EBV-VCA-IgM, and EBV-EA-IgG were conducted using chemiluminescence immunoassay in part of the subjects.

\section{Statistical analysis}

All statistical analyses were performed using SPSS for Windows (version 21.0; IBM Corporation, Armonk, NY, USA) and Graphpad Prism 6. Followup was through September 2018. Progressionfree survival (PFS) was calculated as the duration from initial diagnosis to the first time of progression, relapse from response, death or last follow-up. OS was defined as the time between diagnosis and death or last follow-up. The best cut-off values were determined by X-tile according to OS [12]. Survival curves were carried out using the Kaplan-Meier method and statistical significance was assessed using the two-sided log-rank test. Mann-Whitney $U$ test and Fisher exact test were respectively adopted to conduct correlation analysis between EBV DNA and continuous variables and categorical variables for unpaired samples. The Cox proportional hazards models were established for the estimation of factors in both univariate and multivariate analysis. Multivariate proportional hazards regression was performed with statistically significant factors from the univariate analysis. All statistical tests were twosided, with $P$-values $<0.05$ defined significant. 


\section{Results}

\section{Patient characteristics}

The baseline clinical and pathologic characteristics of 88 patients with MCL involved in this study at admission were presented in Table 1. Among the 88 patients, there were 69 males and 19 females with an $\mathrm{M}$ : $\mathrm{F}$ ratio of $3.6: 1$, presenting a striking male predominance. The median age was 60 years (range, 28 to 81 years) and approximately $51 \%$ of the patients were over 60 years old. Thirteen $(14.8 \%)$ patients had an ECOG score of more than 2. The majority of patients $(94.3 \%)$ were at advanced stage (Ann Arbor stage III-IV) and 57 (64.8\%) patients had BMI. Elevated LDH and $\beta 2-M G$ were shown in 19 $(21.6 \%)$ and $60(68.2 \%)$ patients, respectively. Thirtyeight $(43.2 \%)$ patients were found suffering from B symptoms. The number of patients at low risk (sMIPI score 0-3), intermediate risk (sMIPI score 4-5) and high risk (sMIPI score 6-11) stratified by sMIPI were $40(45.4 \%), 29(33.0 \%)$ and $19(21.6 \%)$, respectively.

Additionally, $27(30.7 \%)$ patients were detected positive for EBV DNA and the median virus titer was $2.05 \times 10^{4}$ copies $/ \mathrm{mL}$ (range, $8.20 \times 10^{2}$ to $4.14 \times 10^{5}$ copies $/ \mathrm{mL}$ ). However, pretreatment results of antiEBV antibody tests of 28 patients were obtained. Our results showed that 12 patients $(42.9 \%)$ had serological evidence of prior EBV-infection (EBNAIgG-positive and EBV-VCA-IgG-positive). Nine patients $(32.1 \%)$ were at the late stage of infection (EBNA-IgG-positive, EBV-VCA-IgG-positive and EBV-EA-IgG-positive) while six patients (21.4\%) were at the early stage of infection (EBV-VCA-IgG-positive, EBV-VCA-IgM-positive and EBV-EA-IgG-positive). Furthermore, one patient $(3.6 \%)$ was found with the positive of EBNA-IgG, EBV-VCA-IgG, EBV-EA-IgG and EBV-VCA-IgM.

Table 1. Clinical characteristics with the analysis of pretreatment EBV DNA in 88 patients with mantle cell lymphoma

\begin{tabular}{lllll}
\hline Characteristic & $\begin{array}{l}\text { No. }(\%) \\
(\mathrm{n}=88)\end{array}$ & $\begin{array}{l}\text { EBV DNA- } \\
\text { positivity }(\mathrm{n}=27)\end{array}$ & $\begin{array}{l}\text { EBV DNA- } \\
\text { negativity }(\mathrm{n}=61)\end{array}$ & $P$-value \\
\hline Male sex & $69(78.4)$ & $25(92.6)$ & $44(72.1)$ & $\mathbf{0 . 0 4 7}$ \\
B symptoms & $38(43.2)$ & $13(48.1)$ & $25(41.0)$ & 0.642 \\
Age $>60$ yr & $45(51.1)$ & $18(66.7)$ & $27(44.3)$ & 0.066 \\
ECOG 2-4 & $13(14.8)$ & $6(22.2)$ & $7(11.5)$ & 0.207 \\
Stage III-IV & $83(94.3)$ & $27(100.0)$ & $56(91.8)$ & 0.318 \\
LDH $>$ ULN & $19(21.6)$ & $8(29.6)$ & $11(18.0)$ & 0.265 \\
WBC $>6.7 \times 10^{9} / \mathrm{L}$ & $50(56.8)$ & $20(74.1)$ & $30(49.2)$ & $\mathbf{0 . 0 3 7}$ \\
BMI & $57(64.8)$ & $19(70.4)$ & $38(62.3)$ & 0.629 \\
$\beta 2-M G>$ ULN & $60(68.2)$ & $22(81.5)$ & $38(62.3)$ & 0.180 \\
sMIPI score 4-11 & $48(54.5)$ & $18(66.7)$ & $30(49.2)$ & 0.077 \\
AMC $>0.5 \times 10^{9} / \mathrm{L}$ & $42(47.7)$ & $17(63.0)$ & $25(41.0)$ & 0.067 \\
EBV DNA-positivity & $27(30.7)$ & - & - & - \\
\hline
\end{tabular}

ECOG, Eastern Cooperative Oncology Group; LDH, serum lactate dehydrogenase; ULN, upper limits of normal; WBC, white blood cells; BMI, bone marrow

involvement; $\beta 2-\mathrm{MG}$, serum beta-2 microglobulin level; sMIPI, simplified mantle cell lymphoma International Prognostic Index; AMC, absolute monocyte counts; EBV, Epstein-Barr virus.
Associations between EBV DNA and other baseline characteristics

Associations between EBV DNA load and other baseline characteristics were described in Table 1. In our cohort, a statistically evident dominance was identified that high level of EBV DNA copy number was more likely in males $(P=0.047)$ and patients with high WBC $\left(>6.7 \times 10^{9} / \mathrm{L}\right)(P=0.037)$. Moreover, EBV DNA-positive patients seemed to take up a larger proportion in age $>60 \mathrm{yr}$ group $(P=0.066)$, intermediate- to high- risk group $(P=0.077)$, indicating a tendency toward significance. With the use of X-tile plots, the cut-off point at $0.50 \times 10^{9}$ monocyte cells/L yielded the highest difference in OS. Among the 42 patients whose monocyte cell counts exceeded the value, there were $17(40.5 \%)$ patients in EBV DNA-positive group while 25 (59.5\%) patients in EBV DNA-negative group. EBV DNA-positive patients seemed to take up a larger proportion in high AMC group $(P=0.067)$. No significant correlations were observed between EBV DNA load and other factors.

\section{Clinical outcomes for MCL patients}

Generally, we conducted efficacy assessment of each patient with the completion of treatment. Subjects received a median of six courses of immunochemotherapy (range 2-6). Median follow-up was 40 months (range, 9 to 120 months). Patients who achieved CR / CRu and PR accounted for $22.7 \%$ and $48.9 \%$, respectively. Forty-three $(48.9 \%)$ patients progressed and $28(31.8 \%)$ died. For the entire cohort, the median PFS and OS were 32 months (range, 1-90 months) and 70 months (range, 2-116 months). The 3-, 5 -year PFS rate was $38.0 \% \pm 5.2 \%$ and $24.0 \% \pm 6.3 \%$, while the 3-, 5-year OS rate was $58.0 \% \pm 5.6 \%$ and $48.0 \% \pm 6.1 \%$.

\section{Prognostic impacts of pretreatment EBV DNA in MCL}

Not only did PFS estimation differ between the pretreatment EBV DNA-positive and EBV DNAnegative group $(P=0.012)$, but also inferior OS was observed in the pretreatment EBV DNA-positive group than in the EBV DNA-negative group $(P=0.004)$, which was shown in Fig. 1 . The 3-year PFS and OS rate were $18.0 \% \pm 8.4 \%$ and $31.0 \% \pm 9.9 \%$ in EBV DNA-positive group vs. $46.0 \% \pm 6.4 \%$ and $69.0 \% \pm 6.7 \%$ in EBV DNA-negative group.

The univariate and multivariate Cox regression analyses of PFS and OS for potential risk predictors in MCL were summarized in Table 2 and Table 3. Univariate Cox regression analysis demonstrated that B symptoms (HR, 2.01; 95\% CI, 1.07 to 3.78; $P=0.029$ ), LDH > upper limits of normal (ULN) (HR, 2.13; 95\% CI, 1.07 to $4.21 ; P=0.030$ ) and EBV DNA-positivity 
(HR, 2.20; 95\% CI, 1.16 to $4.17 ; P=0.015$ ) were related to inferior PFS. In addition, variables including male sex (HR, 8.62; 95\% CI, 1.17 to 63.66; $P=0.035)$, B symptoms (HR, 5.06; 95\% CI, 2.08 to $12.32 ; P<0.001$ ), $\mathrm{LDH}>\mathrm{ULN}(\mathrm{HR}, 2.53 ; 95 \% \mathrm{CI}, 1.12$ to $5.70 ; P=0.026)$, WBC $>6.7 \times 10^{9} / \mathrm{L}(\mathrm{HR}, 2.69 ; 95 \% \mathrm{CI}, 1.16$ to 6.22; $P=0.021$ ), BMI (HR, 3.87; 95\% CI, 1.32 to 11.33; $P=0.014)$, high sMIPI score (HR, 1.75; 95\% CI, 1.08 to 2.84; $P=0.023)$ and EBV DNA-positivity (HR, 3.04; $95 \%$ CI, 1.38 to $6.69 ; P=0.006$ ) had adverse effects on OS. Based on the univariate cox regression analysis results, sMIPI score and EBV DNA-positivity were put into multivariate analysis. As a result, multivariate Cox regression analysis revealed that EBV DNA-positivity was independent risk factor for both PFS (HR, 2.04; 95\% CI, 1.07 to 3.92; $P=0.031$ ) and OS (HR, 2.68; 95\% CI, 1.20 to $6.00 ; P=0.016$ ).

\section{Subgroup evaluation of EBV DNA in MCL}

We constructed subgroup evaluations in order to accurately identify the influence of pretreatment EBV DNA load on PFS and OS combined with the risk parameters in SMIPI, as is illustrated in Fig. 2, Fig. 3, Fig. 4 and Fig. 5. The OS remained shorter in EBV DNA-positive subjects in subgroups such as sMIPI score $0-5(P=0.003)$ (Fig. 2B), age $>60 \mathrm{yr}(P=0.014)$ (Fig. 3A), ECOG 0-1 $(P=0.001)$ (Fig. 3B), WBC $>6.7 \times 10^{9} / \mathrm{L}$ $(P=0.021)$ (Fig. 3C), LDH $\leq$ ULN $(P=0.004)$ (Fig. 3D). Likewise, patients with EBV DNA positivity displayed inferior PFS in subgroups such as sMIPI score $0-5(P=0.025)$ (Fig. 4B), age $>60 \mathrm{yr}(P=0.012)$ (Fig. 5A), ECOG 0-1 $(P=0.013)$ (Fig. 5B), WBC $>6.7 \times 10^{9} / \mathrm{L}$ $(P=0.049)$ (Fig. 5C), LDH $\leq \mathrm{ULN}(P=0.008)$ (Fig. 5D).

\section{Dynamic changes in EBV DNA load}

Among the 27 patients who were EBV DNA-positive at diagnosis, 17 patients had a

A

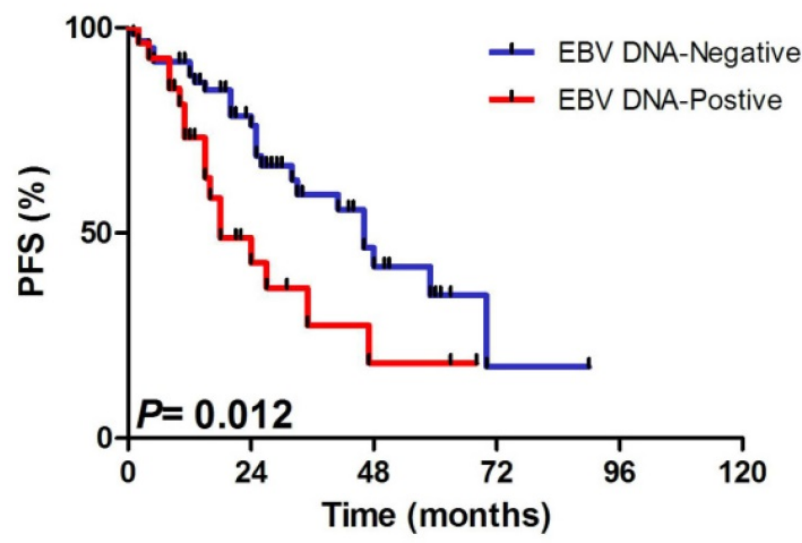

sequential detection of EBV DNA copies during therapy and follow-up. EBV DNA copy numbers were undetectable in 12 patients who had good response to immunochemotherapy though four of them were dead at the end of follow-up deadline. Four patients had a significant increase in EBV DNA copy numbers when they experienced disease progression. One patient was assessed PR after the third cycle of immunochemotherapy regardless of elevated EBV DNA load at this time. However, this patient gave up the following treatment as a result of intolerance to immunochemotherapy due to infection.

Table 2. Univariate Cox regression analysis of PFS and OS in 88 patients with mantle cell lymphoma

\begin{tabular}{lllll}
\hline & \multicolumn{2}{l}{ Univariate analyses $(\mathrm{PFS})$} & \multicolumn{2}{l}{ Univariate analyses (OS) } \\
\hline Characteristic & HR $(95 \% \mathrm{CI})$ & $P$-value & $\mathrm{HR}(95 \% \mathrm{CI})$ & $P$-value \\
Male sex & $1.60(0.70-3.63)$ & 0.262 & $8.62(1.17-63.66)$ & $\mathbf{0 . 0 3 5}$ \\
B symptoms & $2.01(1.07-3.78)$ & $\mathbf{0 . 0 2 9}$ & $5.06(2.08-12.32)$ & $<\mathbf{0 . 0 0 1}$ \\
Age $>60$ yr & $1.31(0.71-2.42)$ & 0.390 & $2.10(0.95-4.63)$ & 0.067 \\
ECOG $2-4$ & $1.73(0.82-3.65)$ & 0.148 & $2.05(0.81-5.16)$ & 0.128 \\
LDH $>$ ULN & $2.13(1.07-4.21)$ & $\mathbf{0 . 0 3 0}$ & $2.53(1.12-5.70)$ & $\mathbf{0 . 0 2 6}$ \\
WBC $>6.7 \times 10^{9} / \mathrm{L}$ & $1.323(0.71-2.46)$ & 0.376 & $2.69(1.16-6.22)$ & $\mathbf{0 . 0 2 1}$ \\
BMI & $1.13(0.58-2.20)$ & 0.712 & $3.87(1.32-11.33)$ & $\mathbf{0 . 0 1 4}$ \\
$\beta 2-M G ~>$ ULN & $1.59(0.73-3.47)$ & 0.244 & $3.11(0.92-10.45)$ & 0.067 \\
sMIPI score & $1.38(0.93-2.06)$ & 0.111 & $1.75(1.08-2.84)$ & $\mathbf{0 . 0 2 3}$ \\
EBV DNA-positivity & $2.20(1.16-4.17)$ & $\mathbf{0 . 0 1 5}$ & $3.04(1.38-6.69)$ & $\mathbf{0 . 0 0 6}$ \\
\hline
\end{tabular}

PFS, progression-free survival; OS, overall survival; HR, hazard ratio; $\mathrm{CI}$, confidence interval; ECOG, Eastern Cooperative Oncology Group; LDH, serum lactate dehydrogenase; ULN, upper limits of normal; WBC, white blood cells; BMI, bone marrow involvement; $\beta 2-\mathrm{MG}$, serum beta-2 microglobulin level; sMIPI, simplified mantle cell lymphoma International Prognostic Index; EBV, Epstein-Barr virus.

Table 3. Multivariate Cox regression analysis of PFS and OS in 88 patients with mantle cell lymphoma

\begin{tabular}{lllll}
\hline & \multicolumn{2}{l}{ Multivariate analyses (PFS) } & \multicolumn{2}{l}{ Multivariate analyses (OS) } \\
\hline Characteristic & HR $(95 \% \mathrm{CI})$ & $P$-value & HR $(95 \% \mathrm{CI})$ & $P$-value \\
sMIPI score & $1.28(0.85-1.91)$ & 0.237 & $1.59(0.98-2.59)$ & 0.062 \\
EBV DNA-positivity & $2.04(1.07-3.92)$ & $\mathbf{0 . 0 3 1}$ & $2.68(1.20-6.00)$ & $\mathbf{0 . 0 1 6}$
\end{tabular}

sMIPI, simplified mantle cell lymphoma International Prognostic Index.

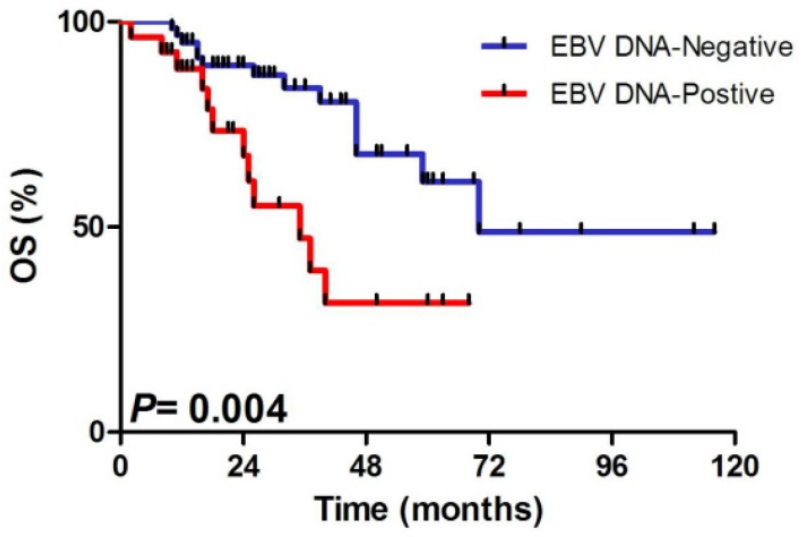

Figure 1. Progressive-free survival (PFS) and overall survival (OS) for 88 patients with the analysis of pretreatment Epstein-Barr virus (EBV) DNA status. 
A

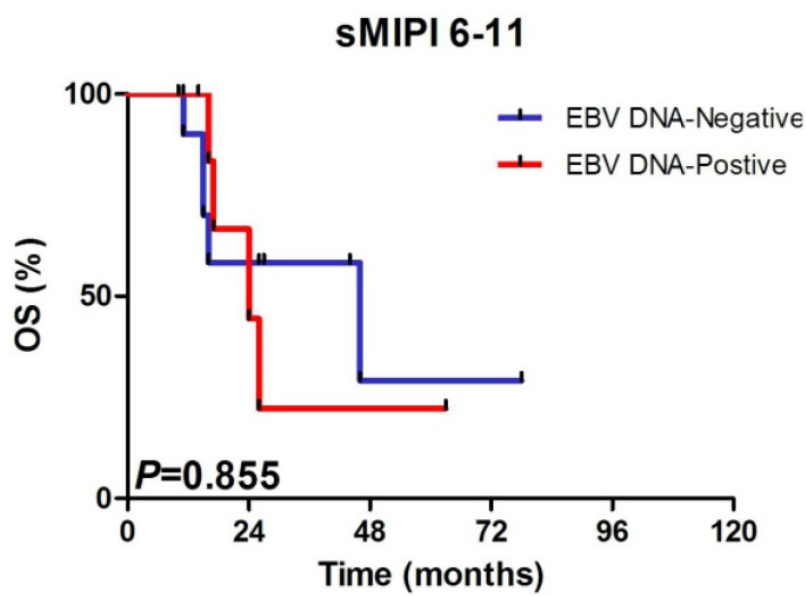

B

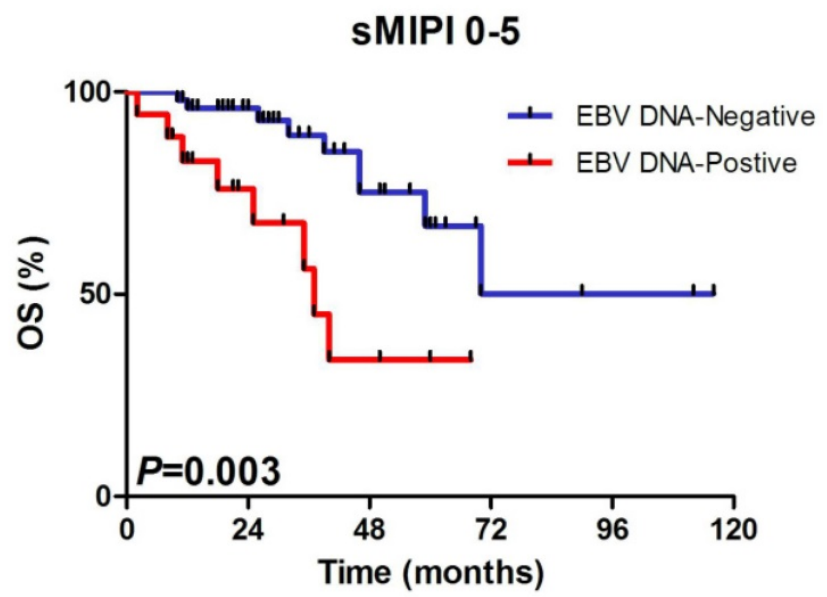

Figure 2. Kaplan-Meier curves of overall survival (OS) for different levels of EBV DNA load according to sMIPI score. sMIPI, simplified mantle cell lymphoma International Prognostic Index

\section{Discussion}

In order to facilitate clinical decisions for MCL, prognostic indexes including sMIPI, Ki-67, TP53, serum $32-\mathrm{MG}$, chromosome karyotype have been assessed to enable risk-adapted treatment strategies [13-17]. With more and more attention to EBV in recent years, EBV DNA load at initial diagnosis has been demonstrated to have an adverse prognostic impact on patients with several lymphomas [4-8]. As is reported in literatures, EBV may activate B cells, stimulate their proliferation, and inhibit their apoptosis. Furthermore, EBV can express latent membrane protein 1 (LMP1), a latent protein expressed, leading to tumor angiogenesis by activating downstream signaling pathways and modulating the expression of cellular genes [18]. However, to the best of our knowledge, it is the first time to interpret the relevance between EBV DNA and MCL though the specific intrinsic mechanism of EBV in MCL is unclear.

In this retrospective research, we checked the prognostic differences between EBV DNA-positive and EBV DNA-negative MCL in small scale and found that the pretreatment EBV DNA-positivity was an effective marker for predicting PFS and OS of MCL patients. Song et al revealed that lymphoma was at an increased risk among individuals with HBV. It was possible that HBV was harbored in non-liver cells, inducing local inflammation, which played a role in the development of cancer. One of the mechanisms of EBV might be the same as that of HBV [19]. Besides, some demonstrated that high level of EBV DNA loads may represent a consequence of the deeper immunosuppression in CLL and AITL $[20,21]$. As is demonstrated in AITL, Liang et al found that EBV
DNA-positive patients were more associated with lower lymphocyte-monocyte ratio (LMR) [8]. Actually, tumor microenvironment and host immunity are significantly associated with outcome in patients with MCL since AMC and absolute CD4+ T cell counts (ACD4C) have been confirmed to be significant predictors of unfavorable OS [22,23]. In the present study, EBV DNA-positive patients tended to have high AMC, which reflected immune-deficient state for MCL patients to some extent, then adding to tumor aggressiveness, though statistical significance was not reached. Therefore, monitoring of AMC, T lymphocyte counts and natural killer cell counts as well as immunoglobulin level might be necessary for MCL patients. In addition, a significant correlation was noted between EBV DNA-positivity and male sex and WBC. Also, patients had high EBV DNA copies tended to be more likely in age $>60 \mathrm{yr}$ group and intermediate- to high- risk group regardless of insignificant difference, in line with the standpoint that latently-EBV-infected B-cells induced sustained telomerase which was associated with an aggressive clinical behavior $[24,25]$. These might account for the relationship between EBV DNA positivity and inferior OS in MCL patients.

In this current study, we only obtained 28 pretreatment results of anti-EBV antibody tests of MCL patients and failed to further explore the relevance between anti-EBV antibody and prognosis and clinical characteristics of MCL. However, according to 24-year experience at a single institution, Huang et al. provided convincing evidence that raised serum EBV EA-IgA and VCA-IgA levels were related to adverse ENKTL profile and correlated with poor treatment response, early relapse, and poor prognosis in patients with ENKTL [26]. It served as a reminder 
that sufficient examinations including anti-EBV antibody tests should be regularly performed for our

\section{A}

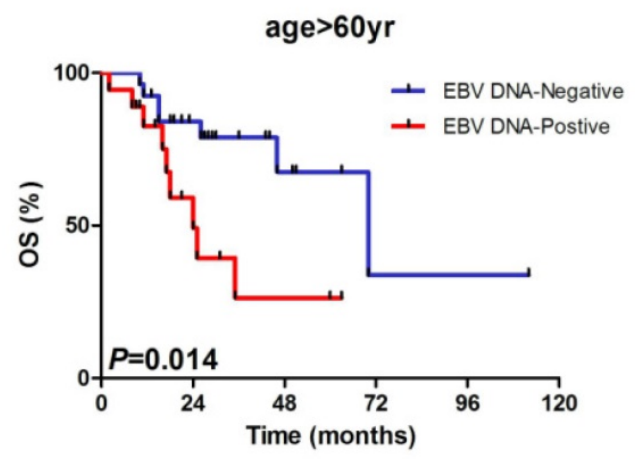

B

ECOG 2-4

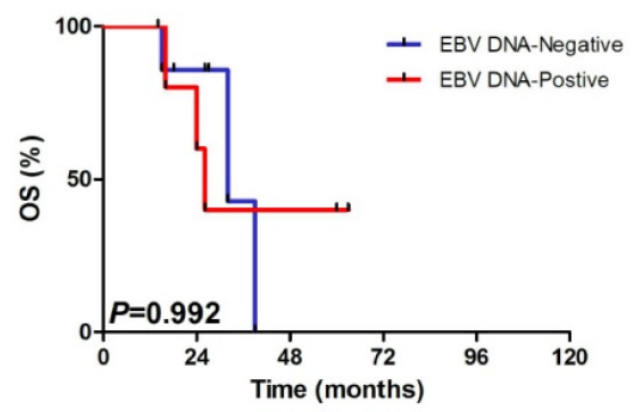

C

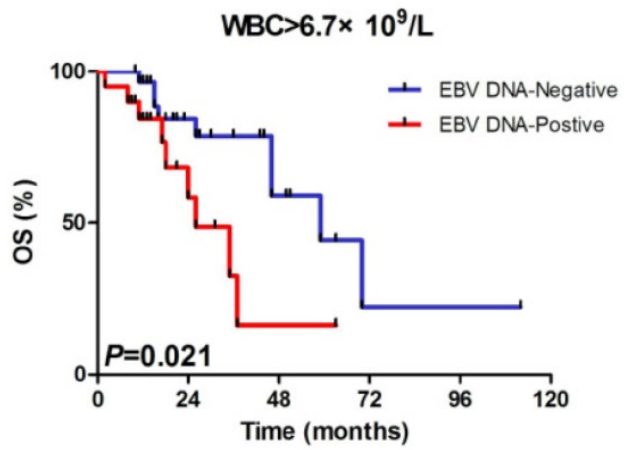

D

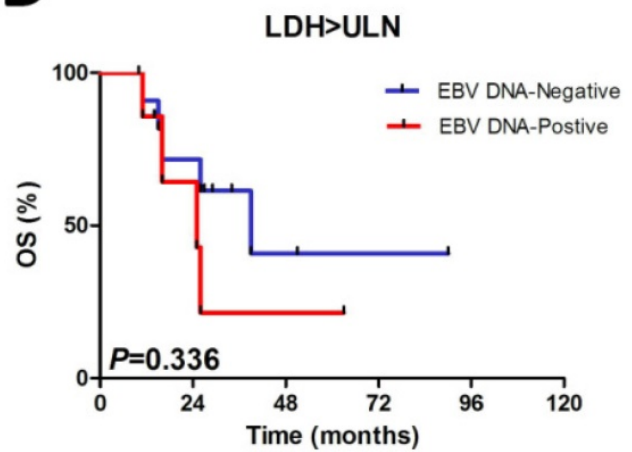

better understanding of MCL.

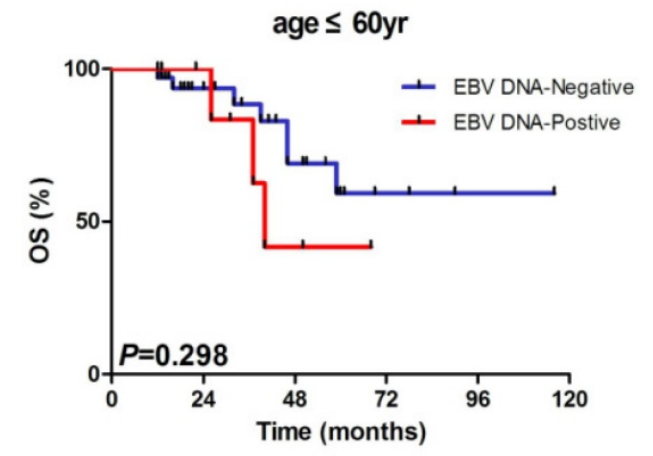

ECOG 0-1

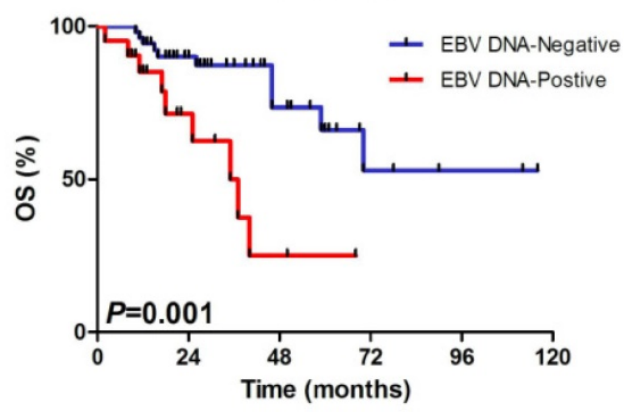

$\mathrm{WBC} \leq 6.7 \times 10^{9} / \mathrm{L}$

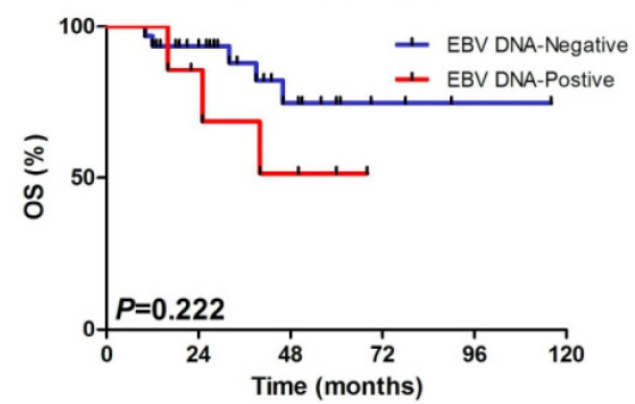

$\mathrm{LDH} \leq \mathrm{ULN}$

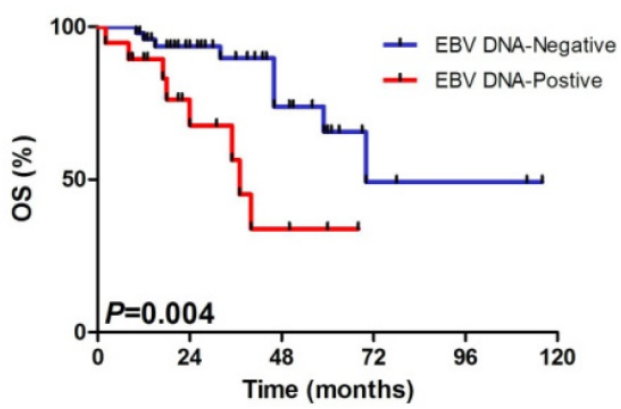

Figure 3. Kaplan-Meier curves of overall survival (OS) for different levels of EBV DNA load stratified by age (A), ECOG (B), WBC (C), LDH (D). ECOG, Eastern Cooperative Oncology Group; WBC, white blood cell counts; LDH, serum lactic dehydrogenase. 
A

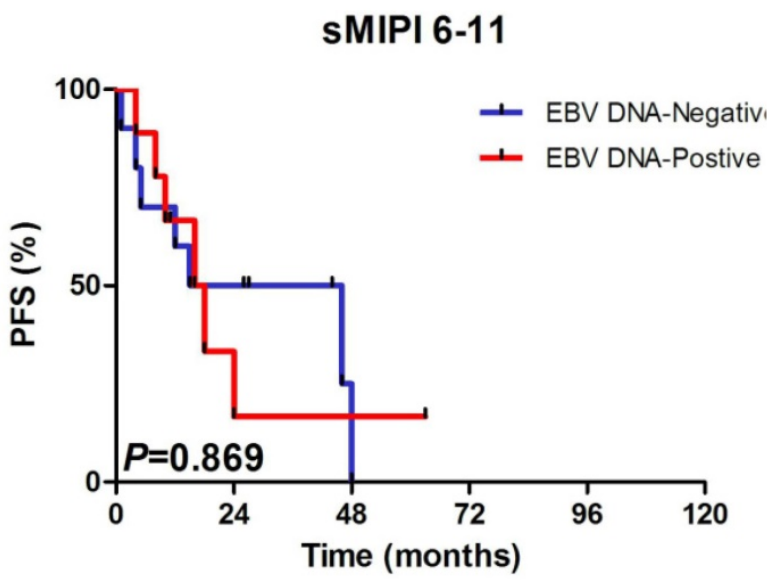

B

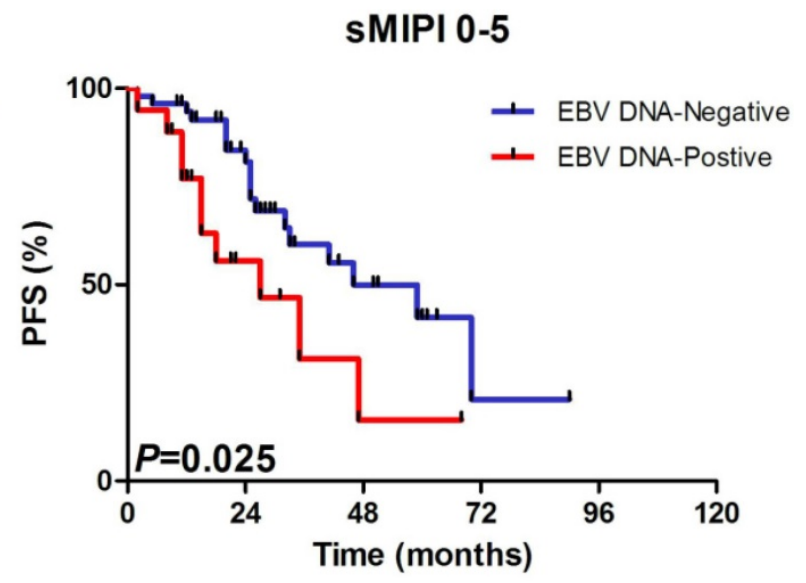

Figure 4. Kaplan-Meier curves of progressive-free survival (PFS) for different levels of EBV DNA load according to sMIPI score. sMIPI, simplified mantle cell lymphoma International Prognostic Index.

As we all known, the International Prognostic Index (IPI), as a traditional model for risk stratification of patients with aggressive non-Hodgkin lymphoma, was discarded for risk stratification of MCL as a result of its weak discriminatory, particularly for the lower risk patients. For better classification, we focused on subgroup analyses and found that the 3-, 5- year PFS and OS rate of EBV DNA-positive group were remarkably lower than EBV DNA-negative group $(18.0 \% \pm 8.4 \%$ and $31.0 \% \pm 9.9 \%$ vs. $46.0 \% \pm 6.4 \%$ and $69.0 \% \pm 6.7 \% ; \quad 18.0 \% \pm 0.0 \%$ and $31 \% \pm 0.0 \%$ vs. $25.0 \% \pm 9.0 \%$ and $55 \% \pm 8.5 \%$, respectively). Beyond that, EBV DNA-positive subjects were noted to have shorter PFS and OS in sMIPI score 0-5, ECOG 0-1 and $\mathrm{LDH} \leq \mathrm{ULN}$ groups, indicating that the survivals of MCL patients with EBV DNA-positivity remained shorter even if he or she was classified as low-risk patients. In addition, patients with EBV DNA-positivity would have much worse PFS and OS if he or she was over $60 \mathrm{yr}$ and had high WBC.

Dynamic detection of EBV DNA changes is of equal important. In this cohort, a phenomenon raised our attention that patients who responded to treatment well were accompanied with decline of EBV DNA load while those who experienced progressed disease were with elevated EBV DNA copy numbers. Of interest, one of the patients was assessed PR after third cycle of treatment with elevated EBV DNA load at this time. Therefore, we come up with some speculations: (1) Whether EBV DNA load could be incorporated into prognostic index together with sMIPI worth further investigation since circulating EBV DNA level was added into the prognostic index for ENKTL (PINK-E); (2) Whether serial monitoring of EBV DNA quantitation after induction chemotherapy should be carried out regularly and formally based on protocol for all patients; (3) Whether appropriate antiviral therapy should be adopted to improve survivals of MCL patients with EBV DNA positivity since we don't have any anti-drug to fight against EBV at present.

It must be admitted that the current study has some limitations. Firstly, due to the retrospective nature, information bias exists. Secondly, on account of the low incidence of MCL, a small cohort of MCL patients was enrolled and the numbers of subjects in some subgroups were small, thus some of the results requiring validation in a larger cohort. Thirdly, Chinese population alone without other populations may limit our ability to discover more information. To sum up, pretreatment EBV DNA copy number was an important prognostic and monitoring marker for MCL patients which might be added into the further prognostic index based on large-scale, multi-central and multi-racial prospective studies for MCL patients.

\section{Acknowledgments}

This study was supported by National Natural Science Foundation of China (81370657, 81470328, 81600130, 81770166, 81720108002), Jiangsu Province's Medical Elite Programme (ZDRCA2016022), Project of National Key Clinical Specialty, National Science \& Technology Pillar Program (2014BAI09B12), Jiangsu Provincial Special Program of Medical Science (BL2014086 and BE2017751) and National Science and Technology Major Project (2017ZX09304032). 
A

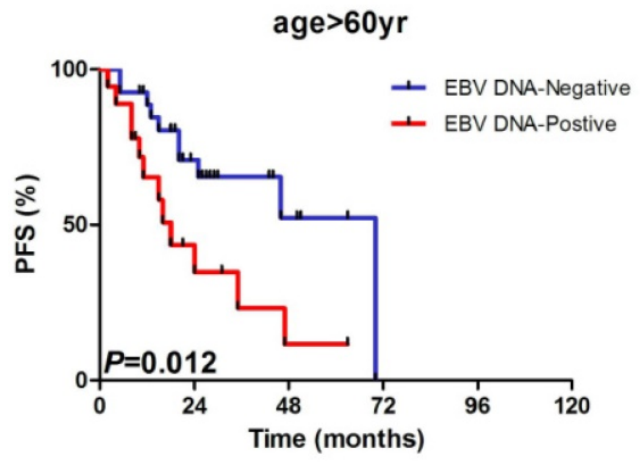

B

ECOG 2-4

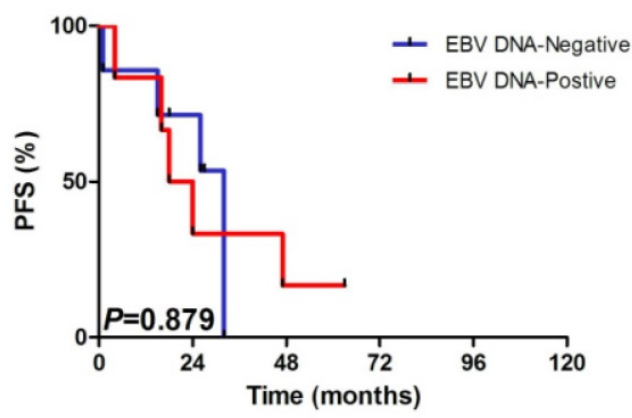

C

$W B C>6.7 \times 10^{9} / \mathrm{L}$

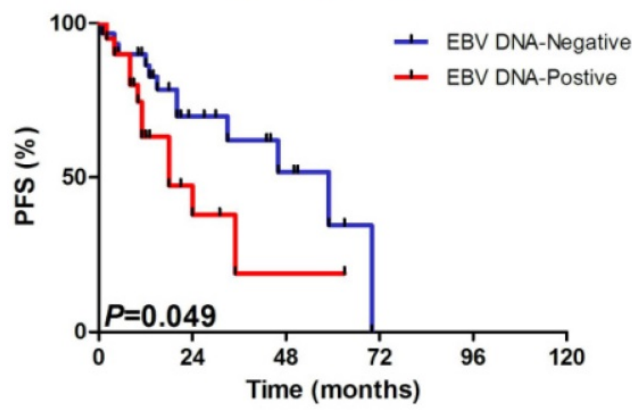

D

LDH>ULN

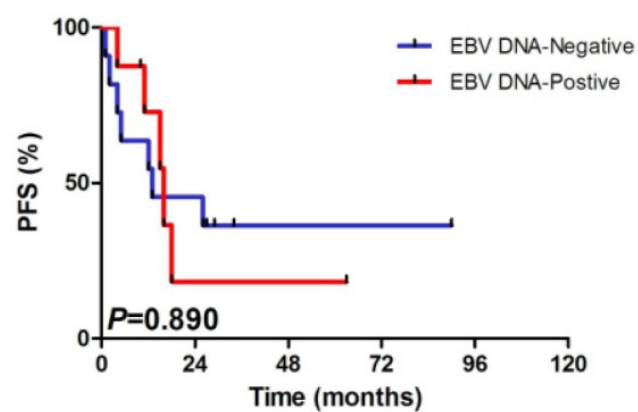

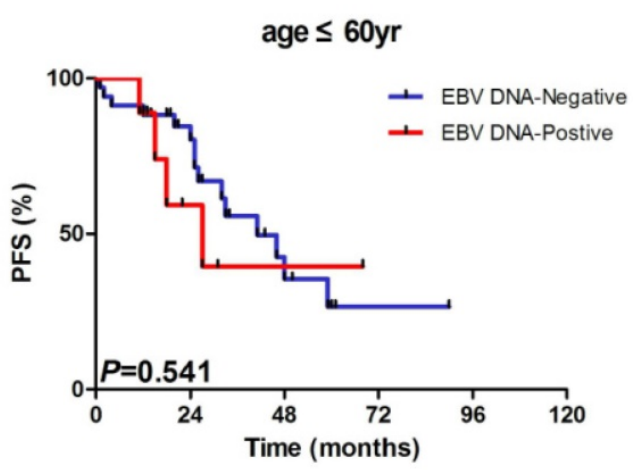

ECOG 0-1

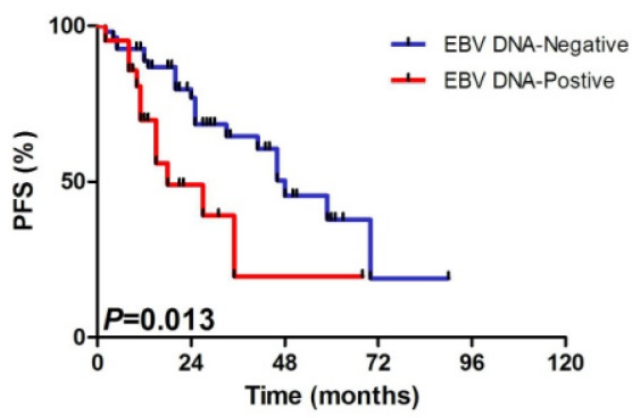

$W B C \leq 6.7 \times 10^{9} / L$

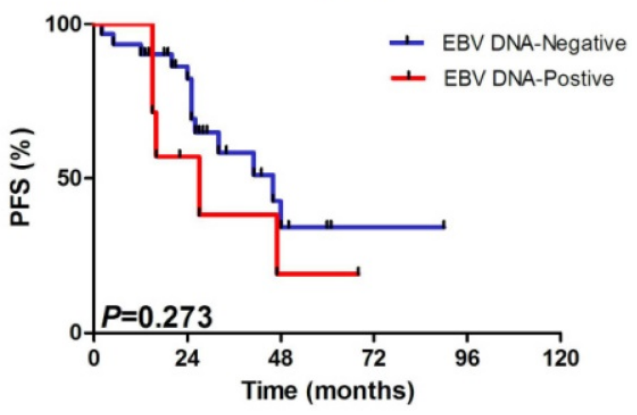

LDH $\leq$ ULN

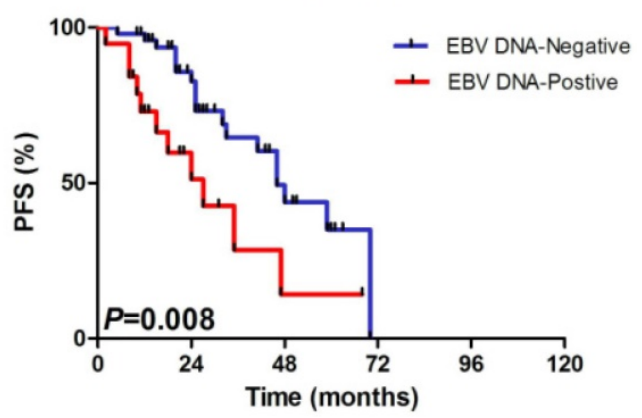

Figure 5. Kaplan-Meier curves of progressive-free survival (PFS) for different levels of EBV DNA load stratified by age (A), ECOG (B), WBC (C), LDH (D). ECOG, Eastern Cooperative Oncology Group; WBC, white blood cell counts; LDH, serum lactic dehydrogenase. 


\section{Competing Interests}

The authors have declared that no competing interest exists.

\section{References}

1. Vose JM. Mantle cell lymphoma: 2017 update on diagnosis, risk-stratification, and clinical management. Am J Hematol. 2017; 92: 806-13.

2. Herrmann A, Hoster E, Zwingers T, et al. Improvement of overall survival in advanced stage mantle cell lymphoma. J Clin Oncol. 2009; 27: 511-8.

3. Kanakry JA, Hegde AM, Durand CM, et al. The clinical significance of EBV DNA in the plasma and peripheral blood mononuclear cells of patients with or without EBV diseases. Blood. 2016; 127: 2007-17.

4. Liang JH, Gao R, Xia Y, et al. Prognostic impact of Epstein-Barr Virus (EBV)-DNA copy number at diagnosis in chronic lymphocytic leukemia. Oncotarget. 2016; 7: 2135-42.

5. Park JH, Yoon DH, Kim S, et al. Pretreatment whole blood Epstein-Barr virus-DNA is a significant prognostic marker in patients with Hodgkin lymphoma. Ann Hematol. 2016; 95: 801-8.

6. Chen R, Wang C, Zhou Y, Wen B. Prognostic implications of circulating Epstein-Barr virus DNA for extranodal natural killer/T-cell lymphoma, nasal type: a meta-analysis. Cancer Manag Res. 2018; 10: 2183-92.

7. Okamoto A, Yanada M, Miura H, et al. Prognostic significance of Epstein-Barr virus DNA detection in pretreatment serum in diffuse large B-cell lymphoma. Cancer Sci. 2015; 106:1576-81.

8. Liang JH, Lu L, Zhu HY, et al. The Prognostic Role of Circulating Epstein-Barr Virus DNA Copy Number in Angioimmunoblastic T-Cell Lymphoma Treated with Dose-Adjusted EPOCH. Cancer Res Treat. 2019; 51: 150-157.

9. Jin Z, Teramoto N, Yoshino $\mathrm{T}$, et al. Characterization of Epstein-Barr Virus-Infected Mantle Cell Lymphoma Lines. Acta Med Okayama. 2000; 54: 193-200.

10. Swerdlow SH, Campo E, Harris NL, et al. World Health Organization Classication of Tumours of Haematopoietic and Lymphoid Tissues. Lyon: IARC Press. 2008.

11. Cheson BD, Horning SJ, Coiffier B, et al. Report of an international workshop to standardize response criteria for non-Hodgkin's lymphomas. NCI Sponsored International Working Group. J Clin Onco. 1999; 17:1244-52.

12. Camp RL, Dolled-Filhart M, Rimm DL. X-tile: a new bio-informatics tool for biomarker assessment and outcome-based cut-point optimization. Clin Cancer Res. 2004; 10:7252-9.

13. Hoster E, Dreyling M, Klapper W, et al. A new prognostic index (MIPI) for patients with advanced-stage mantle cell lymphoma. Blood. 2008; 111: 558-65.

14. Klapper W, Hoster E, Determann O, et al. Ki-67 as a prognostic marker in mantle cell lymphoma-consensus guidelines of the pathology panel of the European MCL Network. J Hematop. 2009; 2: 103-11.

15. Aukema SM, Hoster E, Rosenwald A, et al. Expression of TP53 is associated with the outcome of MCL independent of MIPI and Ki-67 in trials of the European MCL Network. Blood. 2018; 131: 417-420.

16. Yoo C, Yoon DH, Kim S, et al. Serum beta-2 microglobulin as a prognostic biomarker in patients with mantle cell lymphoma. Hematol Oncol. 2016; 34: 22-7.

17. Sarkozy C, Terre C, Jardin F, et al. Complex karyotype in mantle cell lymphoma is a strong prognostic factor for the time to treatment and overall survival, independent of the MCL international prognostic index. Genes Chromosomes Cancer. 2014; 53: 106-16.

18. Koh YW, Han JH, Yoon DH, Suh C, Huh J. Epstein-Barr virus positivity is associated with angiogenesis in, and poorer survival of, patients receiving standard treatment for classical Hodgkin's lymphoma. Hematol Oncol. 2018; 36: $182-188$.

19. Song C, Lv J, Liu Y, et al. Associations Between Hepatitis B Virus Infection and Risk of All Cancer Types. JAMA Netw Open. 2019 ; 2: e195718.

20. Visco C, Falisi E, Young KH, et al. Epstein-Barr virus DNA load in chronic lymphocytic leukemia is an independent predictor of clinical course and survival. Oncotarget. 2015; 6: 18653-63.

21. Zhou Y, Attygalle AD, Chuang SS, et al. Angioimmunoblastic T-cell lymphoma: histological progression associates with EBV and HHV6B viral load. Br J Haematol. 2007;138: 44-53.

22. von Hohenstaufen KA, Conconi A, de Campos CP, et al. Prognostic impact of monocyte count at presentation in mantle cell lymphoma. Br J Haematol. 2013; 162: $465-73$

23. Zhang $\mathrm{XY}, \mathrm{Xu}$ J, Zhu HY, et al. Negative prognostic impact of low absolute CD4+ T cell counts in peripheral blood in mantle cell lymphoma. Cancer Sci. 2016; 107:1471-1476

24. Terrin L, Dal Col J, Rampazzo E, et al. Latent membrane protein 1 of Epstein-Barr virus activates the hTERT promoter and enhances telomerase activity in B lymphocytes. J Virol. 2008; 82: 10175-10187.

25. Rampazzo E, Bonaldi L, Trentin L, et al. Telomere length and telomerase levels delineate subgroups of B-cell chronic lymphocytic leukemia with different biological characteristics and clinical outcomes. Haematologica. 2012; 97: 5663.

26. Huang Y, Rao H, Yan S, et al. Serum EBV EA-IgA and VCA-IgA antibodies can be used for risk group stratification and prognostic prediction in extranodal
NK/T cell lymphoma: 24-year experience at a single institution. Ann Hematol. 2017; 96: 1331-1342. 